\title{
Herpes Simplex Virus Type 1 Promoted the Possibility of Leukemia Caused by L6565 Murine Leukemia Virus
}

\author{
W. Chen, Z.Q. Yang", Y.N. Li and J.J. Liu \\ State Key Laboratory of Virology, Institute of Medical Virology, School of Medicine, Wuhan University, Wuhan, \\ 430071, China
}

\begin{abstract}
Herpes Simplex Virus Type 1 (HSV-1) is a virus commonly existent in nature. Others reported that herpesvirus DNA probably activates type C virus in vitro. L6565 Murine Leukemia Virus (L6565 MuLV) is a kind of virus inducing the murine Leukemia. The main aim of the study was to explore the activation of HSV-1 to the pathogenicity of L6565 MuLV in vivo. Suckling mice were inoculated with either L6565 MuLV \& inactivated HSV-1, or L6565 MuLV alone. In order to determine the infection of the mice, HE staining and RT-PCR techniques were applied. The result shows that the L6565 MuLV RNA was detected in $8^{\text {th }}$ week in the mice of MuLV plus HSV-1 group, while delayed to $10^{\text {th }}$ week in the L6565 MuLV alone group. The infection rates of the two groups were $74.3 \%$ and $41.7 \%$, respectively. Also, L6565 MuLV RNA was detected in the thymus one week after the inoculation, and it was detected in both thymus and spleen two weeks after inoculation. It is obvious that HSV-1 promoted the infection of L6565 MuLV and increased the incidence rate of murine leukemia induced by $\mathrm{L} 6565 \mathrm{MuLV}$.
\end{abstract}

Keywords: Mouse leukemia, L6565 MuLV, HSV-1.

\section{INTRODUCTION}

Murine Leukemia Virus (MuLVs) is a kind of lentivirus that belongs to the Gammaretrovirus family (i.e., type $\mathrm{C}$ virus) [1]. It can induce a number of different types of lymphomas/leukemia in mice, and the main signs of the disease are lymphopenia and immunity descends [2]. Currently, there is some ongoing research on Moloney MuLV, and Friend MuLV [2-5]. L6565 Murine Leukemia Virus (L6565 $\mathrm{MuLV}$ ) is another kind of MuLVs, which was set up in China by Zhen BF, et al. in Department of Pathophysiology, Shanghai Medical University [6]. It offered a new method to study the pathogenicity of leukemia virus. Then Cheng L, et al. in the same department established a viral L6565 cell clone (L6565 Lymphocytes leukemia cell) [7], which was used extensively in MuLV pathogenicity studies $[8,9]$. It was then proven by us that the cell causes Murine Leukemia [10]. Evaluation of the pathogenicity of L6565 MuLV is based on the L6565 cell clone in this study.

Herpes Simplex Virus Type 1 (HSV-1) is a type of Herpes Simplex Virus that belongs to the Herpesvirus family [1]. It is a kind of virus commonly existent in nature. About ninety percent population is in latent infected. Upon infection, the virus is transported by way of axons in the sensory nerve endings to the neurons of sensory ganglia, where it remains latent, and serves as a latent reservoir for recurrent disease in the case of immunity descends or breakdown. Previous studies on the relationship of Leukemia Virus and HSV-1. ANN L, et al. have reported that herpesvirus DNA probably activates type c virus in vitro [11]. Lasky, et al. also proposed that the expression of retroviral proteins

*Address correspondence to this author at the Institute of Virology, Medical School of Wuhan University/State Key Laboratory of Virology, Wuhan, 430071, China; E-mail: zqyang@whu.edu.cn in Ramos lymphoma lines is enhanced after conversion with Herpesvirus [12]. It is suggested that the pathogenicity of MuLV is perhaps related to HSV. An attempt was made to study if HSV-1 promotes the development of Leukemia induced by L6565 MuLV, in vivo. Whereas others have not reported anything.

\section{MATERIAL AND METHOD}

\section{Cells and Viruses}

The L6565 Lymphocytes leukemia cells, which express L6565 MuLV, are obtained from the Department of Pathophysiology, Shanghai Medical University, China. The cell line was maintained in DMEM containing $10 \%$ fatal bovine serum, cultured at $37^{\circ} \mathrm{C}$ in $5 \% \mathrm{CO} 2$. HSV-1 was propagated in Hep-2 cell line, and inactivated by ultraviolet radiation at $50 \mathrm{~W}, 15 \mathrm{~cm}$ for $10 \mathrm{~min}$ before use. Both viruses are preserved in our institution. The Hep-2 cells were maintained in MEM containing $10 \%$ fatal bovine serum. The Coxsackie's $\mathrm{B} 3$ virus was used as a negative control in RT-PCR. It was also propagated in Hep-2 cell line and preserved in our institute. All the viruses were filtered by $0.45 \mu \mathrm{m}$ Millipore before inoculated into mice.

Mice

Kunming suckling mice, born in $24 \mathrm{~h}$, were used for all experiments in this study. Pregnant mice were obtained from Experimental Animal Center of Wuhan University, which maintained and observed individual cages in the holding room before delivery. Births were timed to the nearest $1 / 2$ day.

Newborn Kunming mice were randomly divided into 3 groups in this experiment, they are L6565 MuLV and HSV-1 co-inoculated group, group L6565 MuLV and group control. $0.2 \mathrm{ml}$ of supernate of L6565 Lymphocytes leukemia cells were injected intraperitoneally, and repeated again the next 
day. 100TCID50 HSV-1 was injected with $0.02 \mathrm{ml}$ in hypodermis once a week for the first 2 weeks to sustain the HSV1 antigen in a persistent latent infection.

\section{Tissue Sampling and Preparation}

Two suckling mice of each group were killed every week until MuLV RNA can no longer be detected in the mice (the 14th week according the RNA detection). 16 mice in the L6565 MuLV group and 20 mice in co-infected group were killed, in total. All the main viscera, such as thymus, spleen, lymph node, liver, kidney, and peripheral blood were taken for further studies. Half of them were fixed in $10 \%$ formalin, embedded in paraffin, and sections were stained with hematoxylin and eosin, and Masson's trichrome. While the other half were immediately frozen at $-70{ }^{\circ} \mathrm{C}$ and later used for RNA detection for the determination of L6565 MuLV.

\section{RNA Isolation and RT-PCR Assay}

For the determination of the L6565 MuLV genes expression, total RNA was extracted from each tissue of mice with TRIzol Reagent (Invitrogen) as described in the manufacturer's protocol. RNA quality was assessed by electrophoresis on $1.5 \%$ agarose gel. And to synthesize cDNA by reverse transcription, 2 ul total RNA, 200 units M-MuLV reverse transcriptase (Promega, USA) and 0.5uM oligo dT primer [(5'-GGCCACGCGTCGACTAGTAC(T)16(A/C/G)-3')] were reacted for $1 \mathrm{~h}$ at $37{ }^{\circ} \mathrm{C}$ in $25 \mathrm{ul}$ reaction mixture containing $1 \mathrm{mM}$ dNTP in first strand buffer according to the manufacturer's instruction. PCR amplification was performed with the following described primers: forward primer 5'-GAGACTGTTGGACCAGGGAA - 3' (sense strand correspond to 2834-2854 genome) and a reverse primer 5'-TTGTCCTGAGATTCCCAT-3'(antisense strand correspond to 3125-3143 genome). RNA of L6565 MuLV suspension and Coxsackie's B3 virus were used as positive and negative controls. The PCR reaction was carried out for 30 cycles: $45 \mathrm{~s}$ at $92{ }^{\circ} \mathrm{C}, 45 \mathrm{~s}$ at $57{ }^{\circ} \mathrm{C}$ and 60 at $72{ }^{\circ} \mathrm{C}$, and last extend at $72^{\circ} \mathrm{C}$ for $5 \mathrm{~min}$. PCR products were analyzed on $1.5 \%$ agarose gels for $1 \mathrm{~h}$ at $80 \mathrm{~V}$.

\section{DNA Isolation and PCR Assay}

Genomic DNA was extracted from each mouse tissue as described by the method below. Tissue homogenates were layered over a $10-\mathrm{ml}$ shelf of $35 \%$ sucrose $(\mathrm{wt} / \mathrm{wt})$ in $10 \mathrm{mM}$ Tris/1 mM EDTA, PH 7.4, and the virus was pelleted at $20,000 \mathrm{rpm}$ for $1 \mathrm{hr}$ in an SW-27 rotor. Wash again and treat with RNAse $\mathrm{A}$ and proteinase $\mathrm{K}$ for $15 \mathrm{~min}$ at $68^{\circ} \mathrm{C}$ and then overnight at $37^{\circ} \mathrm{C}$. Genomic DNA was extracted with phenol:chloroform(1:1) twice before use. The isolate was detected by NA Analysator (Eppendorf) to make sure there is no RNA carry over. Then the PCR reaction was carried out in the same conditions as cDNA replication above.

\section{RESULTS}

\section{Infection Comparing}

The number of infection in mice was determined by RNA detection. Viral RNA in the L6565 MuLV plus HSV-1 group is detected 8 weeks after inoculation, while viral RNA in the L6565 MuLV alone infected group is detected 10 weeks after inoculation. In the 14th wk (when there was no L6565
MuLV RNA detected), 35/36 mice remained in the experimental group. Among these mice, the infection rate of L6565MuLV plus HSV-1 group is higher than that of L6565MuLV group (Table 1). Plus, mice that were infected with L6565 MuLV showed signs of infection such as diminished activity and hair stood up.

Table1. L6565 MuLV Infected Condition of Each Group

\begin{tabular}{|c|c|c|c|c|}
\hline Group & $\begin{array}{c}\text { Mice } \\
\text { Number }\end{array}$ & $\begin{array}{c}\text { MuLV Infected } \\
\text { Number }\end{array}$ & $\begin{array}{c}\text { The First Time } \\
\text { of RNA Detected }\end{array}$ & $\begin{array}{c}\text { Infection } \\
\text { Rate (\%) }\end{array}$ \\
\hline \hline L6565MuLV & 36 & 15 & $10 \mathrm{wk}$ & 41.7 \\
\hline $\begin{array}{c}\text { L6565MuL } \\
\text { V+HSV-1 }\end{array}$ & 35 & 26 & $8 \mathrm{wk}$ & $74.3 *$ \\
\hline normal control & 21 & 0 & - & 0.0 \\
\hline
\end{tabular}

\section{Morphology Comparing}

One mouse in the L6565 MuLV plus HSV-1 group developed leukemia, and had greatly enlarged thymus, spleen, liver and lymph nodes. The other mice were in the early phase of leukemia. These include all mice in L6565 MuLV group, which showed a slightly/moderately enlarged spleen and whitened liver. Peripheral lymph nodes and thymuses of these mice were slightly enlarged even at the advanced stage of the disease. The heart, lungs and kidneys of all the mice did not show any obvious changes. Fig. (1) shows the changes of the spleens in erythroleukemia.

(A)

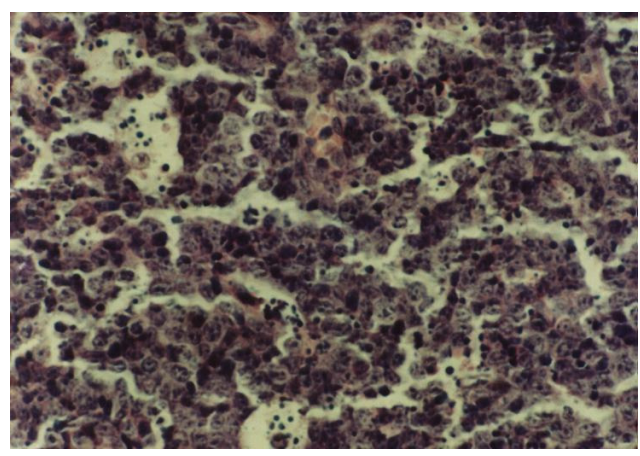

(B)

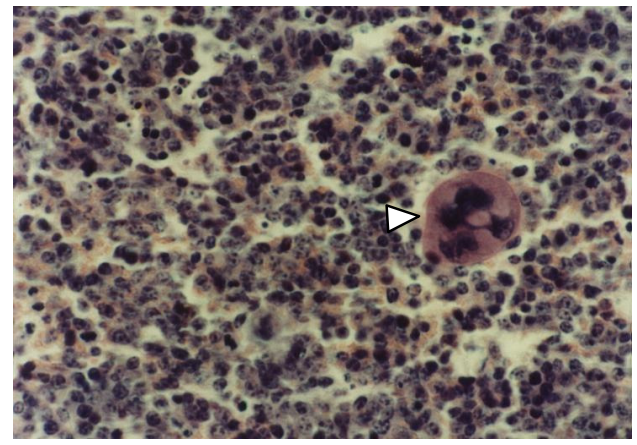

Fig. (1). Spleens of a normal mouse (A: HE x 100) and a mouse with erythroleukemia (B: HE x 100): (A) megakaryocyte $(\Delta)$ is showed in the erythroleukemia tissue slice (B). 


\section{L6565 MuLV RNA and DNA Detection}

In the L6565MuLV plus HSV-1 group, the L6565 MuLV RNA could be detected as early as 1 st week after inoculation and as late as 14 weeks after inoculation. Viral RNA could be detected earlier in mice of the L6565MuLV plus HSV-1 group than in mice of the L6565 MuLV group. The data is shown in Table 2 and Fig. (2). Also, the amplification of RNA and DNA reveals the same amplification fragment (310bp), shown in Fig. (3).

Table 2. The Earliest Time of Each Viscera L6565 MuLV RNA Detected (wk)

\begin{tabular}{|c|c|c|c|c|}
\hline L6565 MuLV RNA & Thymus & Spleen & $\begin{array}{c}\text { Peripheral } \\
\text { Blood }\end{array}$ & Others \\
\hline \hline L6565 MuLV group & 2 & 2 & 3 & 8 \\
\hline $\begin{array}{c}\text { L6565 MuLV plus } \\
\text { HSV-1 group }\end{array}$ & 1 & 2 & 2 & 6 \\
\hline Control group & 0 & 0 & 0 & 0 \\
\hline
\end{tabular}

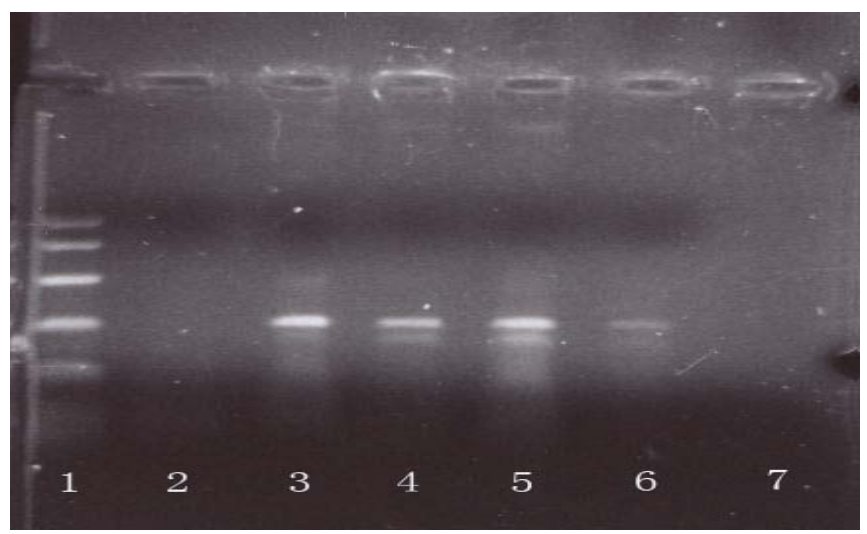

Fig. (2). Detection of L6565 MuLV RNA in the viscera of L6565 MuLV infected mice by RT-PCR: 1. DNA marker; 2. DDW; 3. L6565 Lymphocytes leukemia cell RNA; 4. mouse spleen; 5. mouse lymphoid node; 6 . peripheral blood; 7. CBV3.

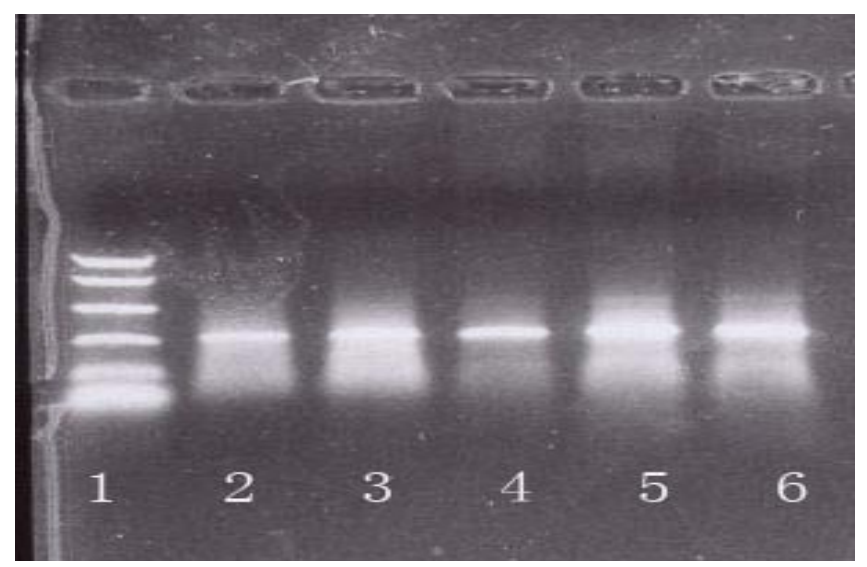

Fig. (3). Comparison of mice RNA and DNA by PCR: 1. DNA marker; 2. L6565 Lymphocytes leukemia cell RNA; 3. L6565 Lymphocytes leukemia cell DNA; 4. peripheral blood DNA; 5. lymphoid node DNA; 6 . spleen DNA.

\section{DISCUSSION}

The study reported here not only confirmed and expanded upon a previous study (Zheng et al. [6]), in which inoculation of L6565 MuLV was found to result in persistent retrovirus infection in kunming suckling mice, but also indicated that HSV-1 possess the ability to activate the pathogenicity of L6565 MuLV in vivo under the conditions used. Other studies have reported that HSV-1, HSV-2 and their DNA or TK-gene-containing cloned sequence all can activate C-type murine retrovirus in vitro [14-16]. Moreover, Liu etc. of our institute have proven that HSV-2 can activate L6565 MuLV [13]. All studies demonstrate that the ability to activate the pathophysiology of L6565 MuLV is a common property shared by all Herpesviruses.

\section{Pathogenicity}

The L6565 Leukemia mice model was established by Zheng BF etc. in Department of Pathophysiology, Shanghai Medical University, in 1965 [6]. It is a utility model to study the etiopathogenesis and pathogenesis of leukemia induced by L6565 MLV. They defined L6565 MuLV as the pathogenic virus for the model, but yet to study the pathogenicity of the virus. We expanded the L6565 MuLV mice model by using L6565 lymphocyte leukemia cells and reported the pathogenicity of the virus [10].

First, our results proved again that $\mathrm{L} 6565 \mathrm{MuLV}$ is a kind of Gammaretroviruses of Retroviridac homologous with Moloney MuLV. We designed a couple of primers based on the conservative nucleotide sequence of the Pol gene in the Moloney MuLV genome [17]. By RT-PCR, we successfully obtained the amplification fragment of L6565 MuLV RNA in both the leukemia cell lines and the tissues of infected mice.

Second, there are two forms of L6565 MuLV that exist in leukemia cells and infected mice: L6565 MuLV RNA and integrated proviral DNA. The DNA isolated from L6565 MuLV infected mice was also detected by PCR in the same conditions as RNA amplification. Both show the same amplification fragment.

Third, similar to Moloney MuLV, L6565 MuLV RNA was first detected in the thymus and spleen, then followed by peripheral blood, liver, kidney and other viscera. By molecular hybridization and PCR technique, previous research shows that MuLV RNA was first detected in bone marrow tissue, spleen and thymus $[2,3]$, and bone marrow multipotent stem cell is the target cell of murine leukemic virus infection [18]. Others reported that onc-genes in leukemic mice, such as cmyc and c-fos, are over-expressed [19] and viral nucleate inserts itself into a region near the cellular proto-oncogene [20]. So we propose that L6565MuLV may be infect lymphoid stem cells in thymus and spleen first, and then insert itself into a place near the cellular proto-oncogene in the form of proviral DNA. Consequently, this activates the expression of those onc-genes and turn lymphoid stem cells into leukemic cells. Once reaching a certain number, the leukemic cells flow with blood, encroach main viscera, and Leukemia appears.

\section{Activation by HSV-1}

Our result demonstrates that the delitescence of the HSV1 plus L6565 MuLV co-infected group (10 weeks) is earlier 
than that of the L6565 MuLV infected group ( 8 weeks); the infection rate increased from $41.7 \%$ to $74.3 \%$ with a statistical significant difference $(\mathrm{P}<0.01)$. From the results, we conclude that HSV-1 can promote the pathogenicity of L6565 MuLV to induce Murine Leukemia.

HSV-1 and HSV-2 can activate type C oncornavirus in vitro, which was discovered by Hamper etc. [14]. Further research demonstrated that HSV-1, HSV-2 and their DNA and TK-gene-containing cloned sequence may all activate $\mathrm{C}$ type murine retrovirus [10]. Other research demonstrated that HSV-1 and HSV-2 can induce host cell transformation. This effect can also be seen in certain viral DNA or inactivated virus, which damaged cytochromatin, induced rearrangement of cell genome, and changed cellular phenotype [21]. Our studies confirmed the fact that HSV activates L6565 MuLV in vivo, and we also suppose the mechanisms could be the ways below. First, it is may be the synergistic effect of HSV-1 and MuLV to promote the transformation of lymphoid stem cells into leukemic cells. The more leukemic cells proliferated, the higher the possibility of Leukemia to appear. Second, HSV-1 maybe damages the host genome, promotes the insertion of MuLV provirus, activates the expression of onc-gene, and then transforms the normal lymphocytes into leukemic cells. Third, HSV-1 maybe activates the replication of $\mathrm{L} 6565 \mathrm{MuLV}$, so as to increase the possibility of infection.

\section{REFERENCES}

[1] David MK, Peter MH. Part II-Specific Virus Family, 16Retroviridae. Fields Virology, the 5th edition, 2007.

[2] Tumas K, Overmoyer B, Clevenger CV, et al. Murine leukemia virus infection in immunocompetent adult mice. Virology 1993; 192(1): 1-9.

[3] Subramanian A, Hegde S, Porayette P, et al. Friend virus utilizes the BMP4-dependent stress erythropoiesis pathway to induce erythroleukemia. J Virol 2008; 82: 382-393.

[4] Traister RS, Lynch WP. Reexamination of Amphotropic Murine Leukemia Virus Neurovirulence: Neural Stem Cell-Mediated Microglial Infection Fails to Induce Acute Neurodegeneration. Virology 2002; 293(2): 262-272.

[5] Jacenisch R, Fan H, Croker B, et al. Infection of preimplantation mouse embryos and of newborn mice with leukemia virus: tissue distribution of viral DNA and RNA and leukemogenesis in the adult animal. Cell Biol 1975; 72(10): 4008-4012.

[6] Zheng BF, Shan YF, Chen L, et al. Isolation and characterization of mouse L6565 leukemia virus. Chin Med J 1985; 98: 655-661.

[7] Cheng L, Yin LH, Zhao XY. Progress in the study on establishment and characteristics of viral L6565 cell clone. Chin J Path 2000; 16(7): 667-669.

[8] Jia M, Yang TH, Yao XJ, et al. A neonatal mouse modal of AIDS induced by L6565 murine leukemia virus. Chin Phar Bull 2005; 21(10): 1271-1273.

[9] Jia M, Yang TH, Yao XJ, et al. Antivirus effects of MYLS prescription on L6565 murine leukemin virus. Chin J New Drugs 2007; 16(16): 1269-1272.

[10] Li YN, Yang ZQ, Liu JJ, et al. L6565 Mouse Leukemia induced by L6565 Murine Leukemia Virus. Cirologica Sinica 2001; 16(3): 261-264.

[11] Boyd AL, Derge JG, Hampar B. Activation of endogenous type C virus in BALB/c mouse cells by herpesvirus DNA. Proc Natl Acad Sci 1978; 75(9): 4558-4562.

[12] Lasky RD, Troy FA. Possible DNA-RNA tumor virus interaction in human lymphomas: expression of retroviral proteins in Ramos lymphoma lines is enhanced after conversion with Epstein-Barr virus. Proc Natl Acad Sci 1984; 81: 33-37.

[13] Liu Jianjun, Guo Guangsong, Yang Zhanqiu, et al. Study for interrelationship between human herpes simplex virus type 2 and leukemia. WUJNS 2000; 5(3): 366-372.

[14] Hamper B, Aaronson SA, Derge JG, et al. Activation of an endogenous mouse type-c virus by ultraviolet-irradiated HSV-1and HSV-2. Pro Natl Acad Sci 1976; 73: 646-650.

[15] Boyd AL, Derge JG, Hamper B, et al. Activation of endogenous type-c virus in BALB/C mouse cells by herpesvirus DNA. Pro Natl Acad Sci 1978; 75: 4558-4562.

[16] Boyd AL, Enquist L, George F, et al. Activation of mouse retrovirus by Herpes simplex virus type-1 cloned DNA Fragments. Virology 1980; 103: 228-231

[17] Shinnick TM, Lerner RA, Gregorsutcliffe J. Nucleotide sequence of Moloney murine leukemia virus. Nature 1981; 293: 543-548.

[18] Tumas K, Garret W, Black K, et al. Murine leukemia virus infects early bone marrow progenitors in immunocompetents mice. Virology 1996; 224: 573-575.

[19] Ying Lianhua, Zheng Songguo, Cheng Li, et al. The initial study of the expression of L6565 murine leukemia oncogene. Chin J Path 1997; 13(2): 173-175.

[20] Bundy LM, Ru M, Zheng BF, et al. Biological characterization and melecular cloning of murine c-type retroviruses derived from the TSZ complex from manland china. Virology 1995; 212: 367-382.

[21] Galloway DA, Mcdougall JK. Alterations in the cellular phenotype induced by Herpes Simplex viruses. J Med Virol 1990; 31: 36-42. 